\title{
Utilizing active single-mode fiber injection for speckle nulling in exoplanet characterization
}

N. Klimovich, Y. Xin, D. Mawet, G. Ruane, J.-R. Delorme, et al.

N. Klimovich, Y. Xin, D. Mawet, G. Ruane, J.-R. Delorme, W. Xuan, D. Echeverri, M. Randolph, J. Fucik, J. K. Wallace, J. Wang, G. Vasisht, R. Dekany, B. Mennesson, E. Choquet, E. Serabyn, "Utilizing active singlemode fiber injection for speckle nulling in exoplanet characterization," Proc. SPIE 10400, Techniques and Instrumentation for Detection of Exoplanets VIII, 104000Y (1 September 2017); doi: 10.1117/12.2275173

EviE Event: SPIE Optical Engineering + Applications, 2017, San Diego, California, United States 


\title{
Utilizing active single-mode fiber injection for speckle nulling in exoplanet characterization
}

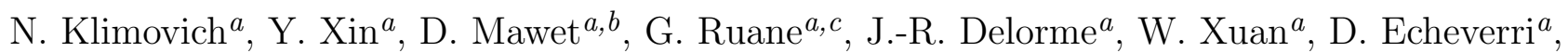

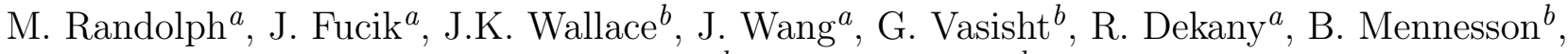 \\ E. Choquet ${ }^{b}$, and E. Serabyn ${ }^{b}$ \\ ${ }^{a}$ Department of Astronomy, California Institute of Technology, Pasadena, CA 91125 \\ ${ }^{b}$ Jet Propulsion Laboratory, California Institute of Technology, Pasadena, CA 91109 \\ ${ }^{c} \mathrm{NSF}$ Astronomy and Astrophysics Postdoctoral Fellow
}

\begin{abstract}
Despite recent advances in high-contrast imaging techniques, high resolution spectroscopy for characterization of exoplanet atmospheres is still limited by our ability to suppress residual starlight speckles at the planet's location. We have demonstrated a new concept for speckle nulling by injecting directly imaged planet light into a single-mode fiber, linking a high-contrast adaptively-corrected coronagraph to a high-resolution spectrograph (diffraction-limited or not). The restrictions on the incident electric field that will couple into the single-mode fiber give the adaptive optics system additional degrees of freedom to suppress the speckle noise on top of destructive interference. We are able to achieve a starlight suppression gains that are an order of magnitude better than conventional techniques in broadband light with minimal planet throughput losses.
\end{abstract}

Keywords: vortex coronagraph, fiber injection unit, speckle nulling, broadband, spectrograph, exoplanet characterization, instrumentation

\section{INTRODUCTION}

Advances in ground-based and space-based instruments and control techniques have enabled astronomers to discover thousands of exoplanets over the past two decades. The vast majority of these detections have been through indirect measurements of the star's luminocity or velocity that offer little information about the exoplanets themselves. ${ }^{1}$ In recent years, many giant exoplanets have been directly imaged through high-contrast imaging techniques, but direct measurements of small, rocky exoplanets in the habitable zone has not yet been achieved with current techniques. If the necessary contrast were to be achieved, high-resolution spectroscopy offers the possibility for detailed characterization of these exoplanet atmospheres.

The combination of high-contrast imaging techniques with high-resolution spectroscopy (here called highdispersion coronagraphy or HDC) is an enticing framework that promises gains beyond the performance boost from the individual techniques ${ }^{2}{ }^{3}$ HDC is perhaps the only method capable of resolving many molecular signatures of life in the atmospheres of exoplanets around nearby M-dwarf stars. ${ }^{4}$ However, significant improvements in starlight suppression are still needed beyond conventional speckle nulling techniques in order to achieve the raw contrast necessary in these measurements.

In this paper, we present the continuation of the work on our concept for feeding a filtered beam of planet light to a high-resolution spectrograph. ${ }^{5}$ This proof-of-concept will be a part of the Keck Planet Imager and Characterizer project (KPIC), a planned upgrade to the W.M. Keck Observatory adaptive optics system and high-contrast instrument suite. ${ }^{6}$ We hope that these on-sky demonstrations can ultimately serve as a basis for similar instruments in future large ground- and space-based facilities: the Thirty Meter Telescope (TMT), the European-Extremely Large Telescope (E-ELT), the Giant Magellan Telescope (GMT), NASA's Habitability Explorer (HabEx), and the Large UV Optical InfraRed (LUVOIR) telescopes.

Techniques and Instrumentation for Detection of Exoplanets VIII, edited by Stuart Shaklan, Proc. of SPIE Vol. 10400, 104000Y · (C) 2017 SPIE · CCC code: 0277-786X/17/\$18 · doi: 10.1117/12.2275173 


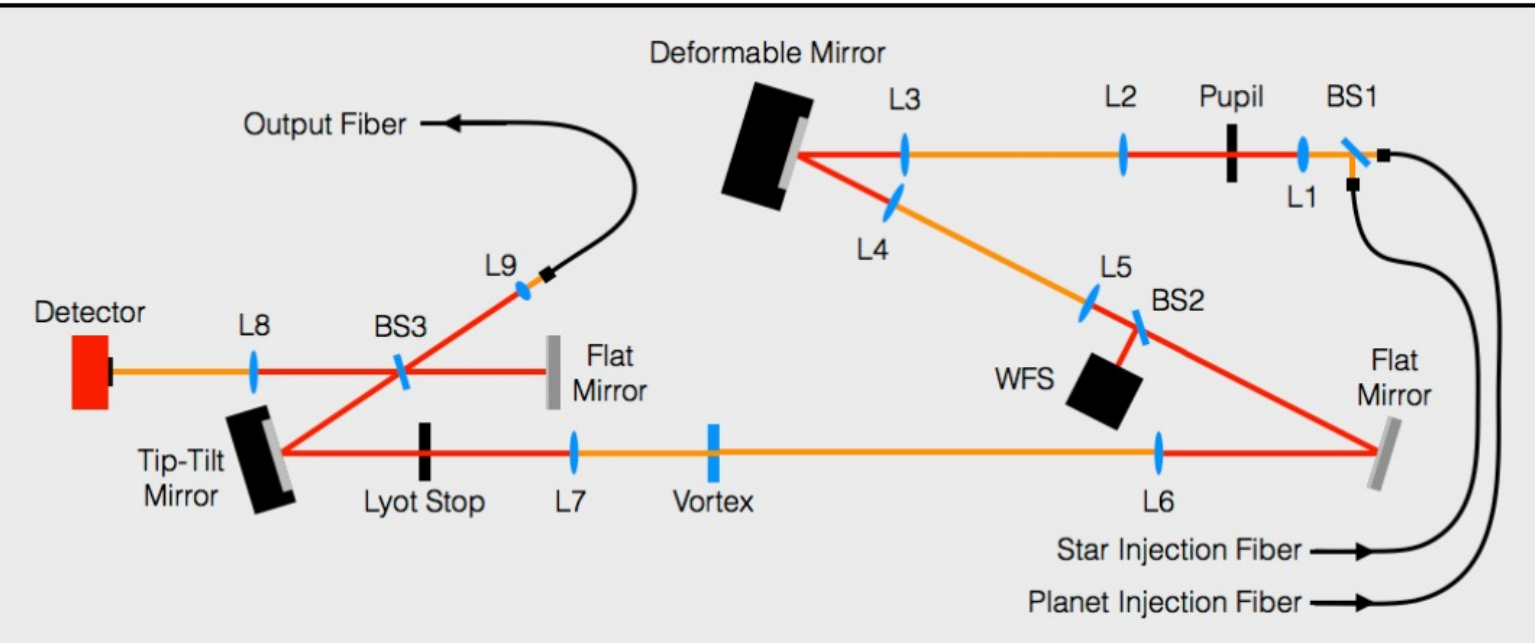

Figure 1. Layout of our laboratory setup, consisting of a star/planet injector, AO system with a deformable mirror (DM) and wavefront sensor (WFS), a vortex coronagraph (vortex), Lyot Stop, Tip-Tilt Mirror, and fiber injection unit. A beamsplitter (BS3) sends a portion of the incident light to a tracking camera (Detector) and light that is retro-fed through the Output Fiber reflects off BS3 and the Flat Mirror onto the Detector for tracking purposes.

\section{EXPERIMENTAL SETUP}

Our laboratory setup consists of a telescope simulator fed by a star-planet injector fiber-fed by an NKT Photonics supercontinuum white light source. Bandpass filters centered around $650 \mathrm{~nm}$ filter the supercontinuum to a $10 \%$ or $20 \%$ bandpass prior to injection into the system. Light from the simulated star and planet is then passed through a Y-junction and the planet light travels through an additional 2 meters of fiber, making it incoherent with light from the simulated star. The telescope simulator is followed by an adaptive optics system consisting of a Boston Micromachines 144-actuator MEMS DM, pellicle beam splitter, and a Shack-Hartmann wavefront sensor (Thorlabs AOK1-UM01).

The AO system is followed by a relay of a focusing lens, focal-plane mask, collimating lens, and Lyot stop. The focal plane mask is a vortex coronagraph, a phase-based coronagraph that rejects light by through a phase singularity at the center of the device that diffracts light outwards to be blocked by a Lyot stop. ${ }^{7}$ This provides an effective inner-working angle of $1.7 \lambda / D$ at which $50 \%$ off-axis transmission is obtained.

After the Lyot stop, the light is directed into a fiber injection unit (FIU) by a computer-controlled three-axis tip-tilt mirror (TTM) with Thorlabs piezo-actuators, allowing for fast, repeatable, high-efficiency coupling of planet light through the single-mode fiber $(\mathrm{SMF})$. In order to locate the position of the fiber in the star-planet system, a 50\%-50\% beamsplitter placed between the TTM and SMF that directs the remaining light onto a Thorlabs CMOS sensor, used as a tracking camera. The SMF is once again directed through a Y-junction where $99 \%$ of the light is sent to a Newport Si photodiode power meter (eventually to be a high-resolution spectrograph) and light is retro-fed through the other arm of the Y-junction to be reinserted into the system. The light is reflected off the beamsplitter, reflected off a corner cube, and reimaged onto the tracking camera. This light allows us to accurately determine the position of the fiber tip relative to the star-planet system.

Due to the lack of atmospheric turbulence and high-performance of the coronagraph, the brightest remaining speckles outside the inner-working-angle are less than $5 \mathrm{nW}$ in power through the fiber when the source is at its maximum power. Since this is only one order of magnitude above the noise floor of our detector, we increase the brightness of the speckle field by introducing optical aberrations into the telescope simulator by placing thin pieces of low-quality glass at the start of the experiment.

\section{FIBER INJECTION UNIT (FIU)}

We have previously proposed a FIU for coupling light from an AO system to a high-resolution spectrograph, in high-dispersion coronagraphy (HDC) systems. ${ }^{5}$ By using a scheme similar to, ${ }^{8}$ we obtain close to theoretical and 
stable planet injection efficiencies in monochromatic light. Here, we propose to further exploit the properties of the SMF for speckle nulling in broadband light.

\subsection{Planet Injection}

The injection efficiency into the SMF, $\eta$, is given by the squared modulous of the overlap integral between the incident electric field, $A(x, y)$, and the fundamental mode of the $\mathrm{SMF}, \mathrm{HE}_{11}:{ }^{9}$

$$
\eta=\frac{\left|\iint \mathrm{HE}_{11}(x, y) A^{*}(x, y) d x d y\right|^{2}}{\iint\left|\mathrm{HE}_{11}(x, y)\right|^{2} d x d y \iint|A(x, y)|^{2} d x d y}
$$

Although the difficulty of light injection into a SMF works as an advantage for starlight suppression, it is disadvantageous because of the planet light throughput loss from the injection efficiency into the SMF. For an ideal circular, unobstructed pupil, the planet point-spread function (PSF) will be an Airy function, which has a theoretical maximum injection efficiency of $81 \%$ for monochromatic light (or broadband light in a perfectly achromatic system). ${ }^{9}$ This maximum efficiency can be increased significantly by apodizing the beam into a Gaussian function to better match the fiber mode. ${ }^{10}$ However, any optical aberrations, chromatic effects, or obstructions will further decrease the maximum injection efficiency in the system. ${ }^{11,12}$

We are able to consistently obtain planet injection efficiencies of 50-55\% through the SMF for $10 \%$ bandpass light by simply adjusting the TTM to direct the planet onto the position of the fiber. These injection efficiencies are significantly lower than the 65-70\% efficiency we are able to obtain with monochromatic light, showing that our system has significant chromatic aberrations. Most of this effect is likely due to the short-focus microscope objective that focuses the light onto the fiber tip.

Although it would be possible to increase the injection efficiency further through apodizing the incident beam and purchasing high-quality achromatic optics, these injection efficiencies are sufficient for purposes of demonstration. Current state-of-the-art AO systems like the Subaru/SCExAO are able to obtain a similar injection efficiency of $50 \%$ when working in H-band, due to the additional residual wavefront error when working on-sky. ${ }^{13,14}$

\subsection{Starlight Suppression}

Passing the light through a single-mode fiber offers several advantages over other methods of directing the imaged planet light to a high-resolution spectrograph.

Firstly, the core size of a single-mode fiber is very small, which shrinks the size of the region in which starlight must be suppressed. Because the DM has a finite number of actuators, there is a limit to the precision with which the wavefront can be manipulated and sinusoidal modes are not truly orthogonal. As a result, starlight suppression obtainable by a DM scales inversely with the size of the dark hole.

Secondly, injection efficiency into a single mode fiber is given by the normalized modulus squared of the overlap integral of the incident electric field at the fiber tip and the fundamental $\mathrm{HE}_{11}$ mode of the fiber. For example, even if the speckle intensity at the fiber tip is non-zero, it is possible that no starlight couples through the fiber if the speckle field is anti-symmetric across the fiber tip. As a result, the DM need only suppress the component of the speckle field that is symmetric across the fiber, with no concern for the anti-symmetric portion, thereby greatly simplifying the suppression problem.

Furthermore, perfect starlight suppression at the spectrograph can be reached even with a non-zero symmetric speckle field across the fiber tip. Second order or even higher term modes that integrate to zero greatly open up the space for allowed solution. 


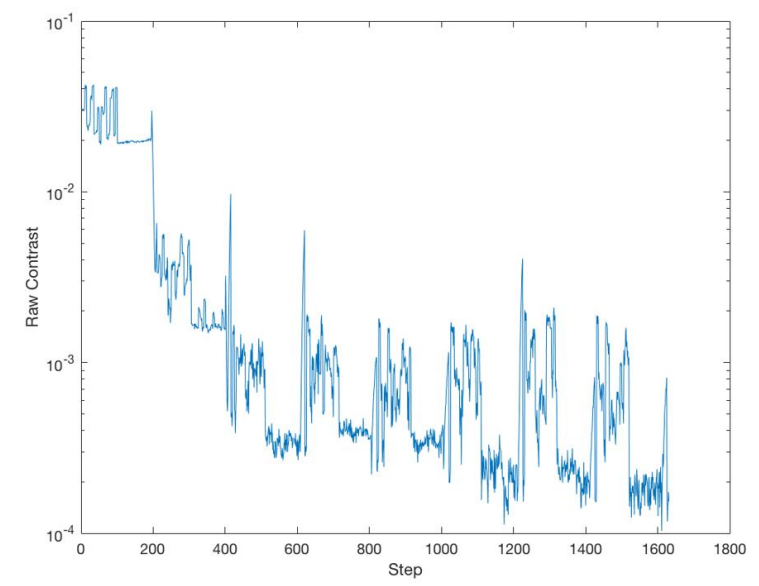

Figure 2. Experimental results of starlight suppression in 610-690 nm (10\% bandpass) light. The raw contrast improves by a factor of 200 over the speckle nulling sequence. Each major 'jump' downwards in starlight suppression occurs when an additional anti-speckle is layered on the DM.

\section{RESULTS}

In order to perform speckle nulling over a broad range of wavelengths, we perform an iterative process of generating anti-speckles at the fiber's location.

A sinusoid with amplitude $h_{0} \ll \lambda$ in the pupil plane will translate into a pair of conjugated anti-speckles in the image plane due to the Fourier transform relationship between the pupil and image plane Malbet1995. In each iteration, we apply a cosine pattern to the DM surface with height

$$
h=\frac{h_{0}}{4}[\cos (2 \pi \vec{\xi} \cdot \vec{r}+\alpha)]
$$

where $h_{0} / 2$ is the maximum surface height ( $h_{0}$ wavefront), $\vec{\xi}$ is the spatial frequency vector and the speckle location in the image plane, $\vec{r}$ is the position vector in the pupil plane, and $\alpha$ is a constant phase offset. The intensity, position, and phase of the speckle are controlled by $h_{0}, \vec{\xi}$, and $\alpha$, respectively.

Our minimization starts by generating a small amplitude $(4 \mathrm{~nm})$ sinusoid with constrained spacial parameters to generate a low-amplitude anti-speckle within a small range of the fiber's known location in the image. The spacial and phase parameters are scanned simulateneously through a generic linear minimization algorithm until the power through the SMF is at a minimum. Afterwards, the amplitude of the anti-speckle is increased to drop the minimum further, while being kept under $20 \mathrm{~nm}$ to preserve planet throughput. Once the phase, spatial, and amplitude for a single sinusoid have been optimized for starlight suppression, the procedure is repeated by layering an additional sinusoid on the DM, following the same procedure.

Results of such a minimization are shown in Fig 2. The noise floor of the detector is generally reached after five to seven sinusoids have been layered on the DM with the amplitude of each sinusoid dropping rapidly after the first two iterations. The anti-speckles produced by this set of iterations is shown in Figure 3. Because the speckle nulling algorithm used is a simple minimization procedure, the solution it finds is normally a combination of destructive interference of the light centered at the fiber tip (first two or three anti-speckles) followed by removal of the more chromatic edges through creating a mismatch between the speckle field and fundamental mode of the SMF. The overall starlight suppression is improved by a factor of 200 in addition to the contrast provided by the coronagraph and AO system for $10 \%$ bandpass light.

Because the simulated planet light is incoherent and much fainter than the simulated starlight, it is mostly unaffected by the minimization procedure. Given that the amplitudes of the deformations on the DM are constrained to be small relative to the wavelength, the PSF of the off-axis planet will remain relatively unchanged. In the minimization shown above, the planet coupling efficiency into the SMF is $84 \%$ of the value that was 


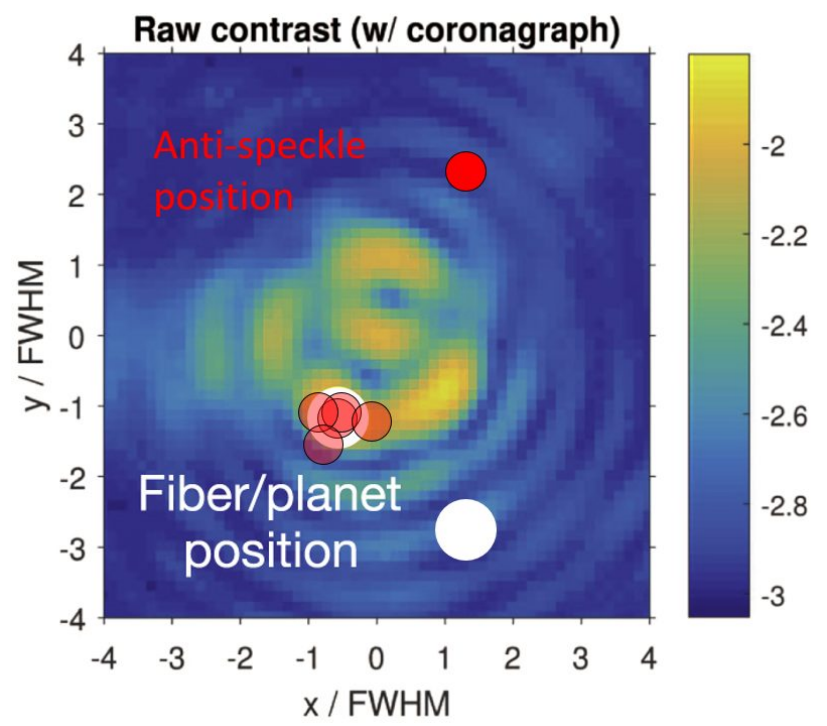

Figure 3. Visualization of the anti-speckles used to minimize starlight at a given planet/fiber position. The first two iterations of the minimization create speckles centered at the fiber location, relying on destructive interference for nulling. Subsequent speckles only affect the electric field at the edges of the fiber, minimizing the symmetric components of the incident field, thereby lowering the starlight injection efficiency.

obtained prior to speckle nulling. If the limiting noise in the measurement is starlight suppression (as opposed to readout or darkcurrent noise in the detector), the improvement in starlight suppression coupled with the loss in planet throughput results in a signal-to-noise improvement in the system of a factor of 12 .

\section{PERSPECTIVES}

The fiber injection unit described here will be the core of the Keck Planet Imager and Characterizer instrument. ${ }^{6}$ These broadband speckle nulling results are a critical part of experimentally demonstrating the effectiveness of the FIU concept that is the fourth and final component of the KPIC design. Before the unit is ready to be integrated, there are still many avenues for starlight suppression we are actively working to explore and improve upon in the near future.

\subsection{Speckle Nulling Algorithm Speed}

The current version of the linear minimization algorithm for finding optimal speckle nulling solutions, while effective, takes on the order of 30 minutes to converge to the final solution. Because our optical system is extremely stable, this speed is more than sufficient for laboratory demonstrations, but is inadequate when attempting to perform real-time wavefront control on a turbulent atmosphere.

Not much work has been done to improve the speed of the algorithm and there are still many angles of approach that could significantly improve the convergence time. In order to compensate for the high noise of the detector, we currently average for a full second for each power reading. A low-noise, high-speed flux measurement could be done at $1 \mathrm{kHz}$, which would instantly improve the speed of the speckle nulling algorithm by an order of magnitude. Furthermore, the linear minimization could be made to converge more quickly by more carefully regulating the step size in the spacial position / phase space of the anti-speckle to more smoothly decrease with time.

Ideally, a linear minimization would be completely unnecessary and speckle nulling would be done by using a technique similar to electric field conjugation (EFC) that minimizes the overlap integral in the injection into the SMF rather than simply the squared amplitude of the electric field. Although there is nothing preventing this in principle, using a SMF for EFC poses the challenge for how to properly sample the spacial dependence of the electric field given the fiber only produces the convolution of the light incident at one point in the focal plane 
with the fiber's fundamental mode. It should be possible to perform this sampling and generate the response matrix of the DM through jittering the fiber location in the image plane, but we are still working on developing the details of this approach.

\subsection{Super-Nyquist Speckle Nulling}

The maximum spacial frequency that can be placed on the DM is limited by the number of actuators, thereby setting a theoretical maximum for the control region for the DM. The number of actuators illuminated on our DM is approximately $11 \mathrm{x} 11$, which means we are limited to roughly 5 cycles in the pupil diameter. Classically, this means we are unable to generate anti-speckles and control the electric field outside of $5 / D$ angular separation.

However, high-frequency spatial features of the DM, such as surface imperfections caused by actuators and cupping effects, can be exploited to generate harmonics that fall outside of this classical control region. ${ }^{15}$ Recent work has shown the possibility of suppressing starlight in this Super-Nyquist regime, which is particularly useful when imaging planets around binary star systems. ${ }^{15}$ We are currently working on testing the effectiveness of these techniques when coupled with a SMF. ${ }^{15}$

\section{CONCLUSION}

In this paper, we presented an innovative fiber injection unit module designed to efficiently couple a highcontrast imaging system (adaptive optics and coronagraph) to a high-resolution spectrograph, enabling highdispersion coronagraphy (HDC) of exoplanets. We have upgraded our FIU prototype to work under more realistic conditions of broadband light and higher wavefront aberrations, continuing to demonstrate the effectiveness of this technology.

We have demonstrated a significant improvement in starlight suppression over a $10 \%$ bandwidth of light through the use of modified speckle nulling techniques combined with the additional mechanisms of starlight suppression offered by a SMF. By ensuring that planet injection efficiency remains high through this process, these techniques promise to provide a significant improvement in signal-to-noise ratio for most conventional exoplanet spectroscopy applications.

Our FIU demonstrator is a prelude to the High Contrast Spectroscopy for Segmented Telescopes Testbed (HSCT-ST) that will allow us to better test this technology through higher quality optics, better wavefront control with multiple DMs, obstructed aperture and segmented telescope simulated, and non-static turbulence generation. This work is the prelude to on-sky scientific demonstrations of the FIU with the KPIC project, which is currently under construction and will begin measurements in 2018.

\section{ACKNOWLEDGMENTS}

The authors would like to acknowledge the financial support of the Heising-Simons foundation.

\section{REFERENCES}

1. B. Bowler, "Imaging extrasolar giant planets," Publications of the Astronomical Society of the Pacific 128, Aug. 2016.

2. P. Riaud and J. Schneider, "Improving Earth-like planets' detection with an ELT: the differential radial velocity experiment," 469, pp. 355-361, July 2007.

3. I. Snellen, R. de Kok, J. L. Birkby, B. Brandl, M. Brogi, C. Keller, M. Kenworthy, H. Schwarz, and R. Stuik, "Combining high-dispersion spectroscopy with high contrast imaging: Probing rocky planets around our nearest neighbors," 576, p. A59, Apr. 2015.

4. J. Wang, D. Mawet, G. Ruane, B. Benneke, and R. Hu, "Observing exoplanets with high dispersion coronagraphy. i. the scientific potential of current and next-generation large ground and space telescopes," 153, 2017.

5. D. Mawet, G. Ruane, W. Xuan, D. Echeverri, N. Klimovich, M. Randolph, J. Fucik, J. Wallace, J. Wang, G. Vasisht, R. Dekany, B. Mennesson, E. Choquet, J.-R. Delorme, and E. Serabyn, "Observing Exoplanets with High-Dispersion Coronagraphy II. Demonstration of an Active Single-mode Fiber Injection Unit," 838, p. 92, Mar. 2017. 
6. D. Mawet, P. Wizinowich, R. Dekany, M. Chun, D. Hall, S. Cetre, O. Guyon, J. K. Wallace, B. Bowler, M. Liu, G. Ruane, E. Serabyn, R. Bartos, J. Wang, G. Vasisht, M. Fitzgerald, A. Skemer, M. Ireland, J. Fucik, J. Fortney, I. Crossfield, R. Hu, and B. Benneke, "Keck planet imager and characterizer: concept and phased implementation," Proc. SPIE 9909, p. 99090D, 2016.

7. D. Mawet, P. Riaud, O. Absil, and J. Surdej, "Annular Groove Phase Mask Coronagraph," 633, pp. 11911200, Nov. 2005.

8. M. M. Colavita, J. K. Wallace, B. E. Hines, Y. Gursel, F. Malbet, D. L. Palmer, X. P. Pan, M. Shao, J. W. Yu, A. F. Boden, P. J. Dumont, J. Gubler, C. D. Koresko, S. R. Kulkarni, B. F. Lane, D. W. Mobley, and G. T. van Belle, "The Palomar Testbed Interferometer," 510, pp. 505-521, Jan. 1999.

9. S. Shaklan and F. Roddier, "Coupling starlight into single-mode fiber optics," 27, pp. 2334-2338, June 1988.

10. N. Jovanovic, F. Martinache, O. Guyon, C. Clergeon, G. Singh, T. Kudo, V. Garrel, K. Newman, D. Doughty, J. Lozi, J. Males, Y. Minowa, Y. Hayano, N. Takato, J. Morino, J. Kuhn, E. Serabyn, B. Norris, P. Tuthill, G. Schworer, P. Stewart, L. Close, E. Huby, G. Perrin, S. Lacour, L. Gauchet, S. Vievard, N. Murakami, F. Oshiyama, N. Baba, T. Matsuo, J. Nishikawa, M. Tamura, O. Lai, F. Marchis, G. Duchêne, T. Kotani, and J. Woillez, "The Subaru Coronagraphic Extreme Adaptive Optics system: enabling high-contrast imaging on solar-system scales," $\mathbf{1 2 7}(955)$, pp. 890-910, 2015.

11. R. E. Wagner and W. J. Tomlinson, "Coupling efficiency of optics in single-mode fiber components," Appl. Opt. 21(15), pp. 2671-2688, 1982.

12. M. Toyoshima, "Maximum fiber coupling efficiency and optimum beam size in the presence of random angular jitter for free-space laser systems and their applications," J. Opt. Soc. Am. A 23(9), pp. 2246-2250, 2006.

13. N. Jovanovic, N. Cvetojevic, C. Schwab, B. Norris, J. Lozi, S. Gross, C. Betters, G. Singh, O. Guyon, F. Martinache, D. Doughty, and P. Tuthill, "Efficiently feeding single-mode fiber photonic spectrographs with an extreme adaptive optics system: on-sky characterization and preliminary spectroscopy," in Society of Photo-Optical Instrumentation Engineers (SPIE) Conference Series, 9908, p. 99080R, Aug. 2016.

14. A. Bechter, J. Crass, R. Ketterer, J. R. Crepp, R. O. Reynolds, E. Bechter, P. Hinz, F. Pedichini, M. Foley, E. Runburg, E. E. Onuma, S. Gaudi, G. Micela, I. Pagano, and C. E. Woodward, "On-sky single-mode fiber coupling measurements at the large binocular telescope," Proc. SPIE 9909, p. 99092X, 2016.

15. S. Thomas, R. Belikov, and E. Bendek, "Techniques for High-contrast Imaging in Multi-star Systems. I. Super-Nyquist Wavefront Control," 810, p. 81, Sept. 2015. 